Revue internationale P.M.E.

Économie et gestion de la petite et moyenne entreprise

Revure

internationale

PME

\title{
Les actions grises dans la théorie des ressources : une compétence cardinale pour la PME?
}

\section{Samuel Grandval et Richard Soparnot}

Volume 16, numéro 3-4, 2003

URI : https://id.erudit.org/iderudit/1008448ar

DOI : https://doi.org/10.7202/1008448ar

Aller au sommaire du numéro

Éditeur(s)

Presses de l’Université du Québec

ISSN

0776-5436 (imprimé)

1918-9699 (numérique)

Découvrir la revue

Citer cette note

Grandval, S. \& Soparnot, R. (2003). Les actions grises dans la théorie des ressources : une compétence cardinale pour la PME? Revue internationale P.M.E., 16(3-4), 121-149. https://doi.org/10.7202/1008448ar
Résumé de l'article

Les actions grises constituent une pratique d'influence qui permet d'atténuer les conditions théoriques et légales de la concurrence. Elles se distinguent du concept de veille stratégique en liant information et action et demandent des ressources spécifiques. Toutefois, leur maîtrise permet-elle de fonder un avantage concurrentiel soutenable ? Nous référant au modèle des ressources et compétences, nous mettons le concept d'actions grises à l'épreuve des critères de l'avantage concurrentiel. L'étude de cinq PME, sélectionnées pour leur maîtrise des actions grises et l'impact décisif engendré, nous amène à conclure que cette compétence confère un avantage concurrentiel soutenable. Notre résultat doit cependant être nuancé. En effet, l'analyse du critère de la longévité amène à préconiser le maintien d'un système de veille traditionnel comme mode d'observation et de surveillance de l'environnement. 


\title{
Note de recherche \\ Les actions grises dans la théorie des ressources: une compétence cardinale pour la PME?
}

\author{
Samuel GRANDVAL \\ ESCEM Tours-Poitiers \\ Richard SOPARNOT \\ Université Évry Val d'Essonne et \\ ESCEM Tours-Poitiers
}

\author{
MOTS CLÉS
}
Actions grises - Avantage concurrentiel - Intelligence économique Ressources - Compétences - Compétence cardinale

\begin{abstract}
RÉSUMÉ
Les actions grises constituent une pratique d'influence qui permet d'atténuer les conditions théoriques et légales de la concurrence. Elles se distinguent du concept de veille stratégique en liant information et action et demandent des ressources spécifiques. Toutefois, leur maîtrise permet-elle de fonder un avantage concurrentiel soutenable? Nous référant au modèle des ressources et compétences, nous mettons le concept d'actions grises à l'épreuve des critères de l'avantage concurrentiel. L'étude de cinq PME, sélectionnées pour leur maîtrise des actions grises et l'impact décisif engendré, nous amène à conclure que cette compétence confère un
\end{abstract}

\section{LES AUTEURS}

Samuel GrandVal détient un doctorat en sciences de gestion et il est professeur de stratégie au groupe ESCEM Tours-Poitiers. Adresse : ESCEM, 1, rue Léo-Délibes, B.P. 0535, 37205 Tours Cédex 3, France. Téléphone : 02477171 71, télécopieur : 02477172 10. Courriel: <sgrandval@escem.fr>.

RICHARD SOPARNOT est enseignant-chercheur à l'Université d'Évry Val d'Essonne et au groupe ESCEM Tours-Poitiers. II est aussi membre du centre Pierre-Naville. Adresse : ESCEM, 1, rue Léo-Délibes, B.P. 0535, 37205 Tours Cédex 3, France. Téléphone : 0247717171 , télécopieur : 02477172 10. Courriel : <rsoparnot@escem.fr>. 
avantage concurrentiel soutenable. Notre résultat doit cependant être nuancé. En effet, l'analyse du critère de la longévité amène à préconiser le maintien d'un système de veille traditionnel comme mode d'observation et de surveillance de l'environnement.

\begin{abstract}
The "grey actions" concept is a managerial method that limits the theoretical and legal conditions of competition. It is apart from business intelligence concept because it links information and action. It is also based on specific resources. Is this practice able to provide with a competitive and sustainable advantage? Refering the RBV theory, we confront the "grey actions" concept with the criteria of competitive advantage. The study of five small and medium size firms, selected for their expertises in "grey actions" and the generated impact, leads us to conclude that this skill provides with a sustainable competitive advantage. However this conclusion should be finely qualified. Indeed the "Iongevity" criteria implies the maintenance of a business intelligence system as a way to observe the environment.
\end{abstract}

\title{
RESUMEN
}

Las "acciones grises » constituyen una practica de influencia al permitir de suavizar las condiciones teoricas y legales de la competencia. Es algo aparte del concepto de la estrategia al vincular informacion y accion y piden recursos especificos. Sin embargo, podemos preguntarnos si su dominio nos permite fundamentar una ventaja competitiva sostenible? Referiendonos al modelo de los recursos y competencias ponemos el concepto de «acciones grises " a la prueba de criterios de ventaja competitiva. El estudio de cinco PME (Pequenas medianas empresas) seleccionadas por su dominio de "las acciones grises» y el impacto decisibo generado nos permite concluir que esta competencia confiere una ventaja competitiva sostenible. Nuestro resultado tiene que ser de hecho de matizado. Savemos que el analisis del criterio de la longevidad nos lleva a preconizar la conservacion de un sistema, tradicional como modo de observacion y de vigilancia del medio ambiente.

\section{ZUSAMMENFASSUNG}

Die Aktionen im Graubereich stellen eine Praxis der Einflussnahme dar, die erlauben, die theoretischen und legalen Bedingungen des Wettbewerbs zu verringern. Sie unterscheiden sich vom strategischen Überwachungskonzept, indem sie Information und Aktion verbindet und spezifische Mittel verlangt.

Erlaubt allerdings ihre Beherrschung wirklich die Begründung eines dauerhaften wettbewerbsfähigen Vorteils? Wir beziehen uns auf das Modell der Ressourcenkompetenzen und unterstellen das Konzept fünf Prüfungskriterien. Die Untersuchung bei fünf ausgewählten KMU veranlasste uns zur Schlussfolgerung, dass die Beherrschung wirklich einen dauerhaften wettbewerbsfähigen Vorteil verleiht. Unser Ergebnis muss jedoch abgestuft werden. In der Tat, ist aber die Analyse des Kriteriums der langen Lebensdauer abhängig von der Aufrechterhaltung eines traditionelles Überwachungssystem, das als Beobachtungs- und Überwachungsmethode der Umwelt eingesetzt wird. 


\section{Introduction}

De la pensée en stratégie semblent se distinguer deux courants théoriques principaux. Spanos et Lioukas (2001) soulignent qu'ils n'en sont pas moins complémentaires. Le premier de ces courants accorde au marché un pouvoir central. La stratégie consiste alors à construire un avantage qui permet de jouer mieux que les concurrents. Pour cette école, les déterminants externes expliquent et orientent les choix stratégiques des firmes. Ce courant, largement initié par les travaux de Porter, correspond selon la terminologie de Martinet (1996) au strategic fit. Le strategic intent émerge dans les années 1980 et constitue un courant théorique aujourd'hui dominant dans la pensée stratégique. Ces années sont caractérisées par une accélération des changements environnementaux et une transformation du jeu concurrentiel affectant les positions acquises par les firmes dominantes. Celles-ci doivent désormais rompre avec les règles qui prévalent dans le secteur pour les reconfigurer à leur avantage et en imposer de nouvelles. Les déterminants internes jouent dans ces conditions un rôle central dans la compétition interentreprises. C'est ainsi que l'approche fondée sur les ressources marque le champ stratégique; elle s'intéresse particulièrement aux mécanismes internes qui permettent à une firme de développer un avantage concurrentiel (Durieux, Girod-Seville et Perret, 2000).

L'intelligence économique a souvent été présentée comme une pratique permettant d'acquérir un avantage concurrentiel. En effet, «L'intelligence économique se définit comme l'ensemble des actions coordonnées de recherche, de traitement, de distribution et de protection de l'information [...] utile aux acteurs économiques en vue de la mise en œuvre de leurs stratégies individuelles et collective » (Martre, 1994). Partant, «L'information utile est celle dont ont besoin les différents niveaux de décision de l'entreprise ou de la collectivité pour élaborer et mettre en œuvre de façon cohérente la stratégie et les tactiques nécessaires à l'atteinte des objectifs définis par l'entreprise dans le but d'améliorer sa position dans son environnement concurrentiel » (Martre, 1994). Ainsi, «l'intelligence économique prolonge les différentes actions de veille et de protection du patrimoine, en intégrant précisément les actions d'influence et les réalités culturelles liées à chaque entreprise, à chaque région » (Clerc, 1995, p. 17). Les actions grises qui visent à atténuer les conditions théoriques et légales de la concurrence constituent une pratique d'influence liant information et action. Par là même, elle fait appel à des ressources spécifiques et constitue une compétence clé. Est-il alors possible d'observer autour des actions grises cette conjonction d'éléments qui fonderaient une compétence distinctive ? En effet, selon Baumard (1997), l'intelligence économique souffre d'un manque de mesure de sa performance par sa nature complexe qui conjugue plusieurs compétences organisationnelles. Le but du présent papier est d'évaluer la portée stratégique des actions grises de la théorie des ressources et compétences. 
Nous définirons donc le concept d'actions grises. Puis, nous exposerons le modèle des ressources et des compétences (MRC). En particulier, nous cherchons à comprendre sur quoi repose l'avantage concurrentiel. Ce cadre nous permettra, dans un second temps, à partir de cinq études de cas, de mettre l'intelligence économique à l'épreuve du $\mathrm{MRC}^{1}$. Ainsi, à partir de la notion d'impact décisif, nous pourrons évaluer les conditions dans lesquelles l'intelligence économique constitue une compétence distinctive fondant un avantage concurrentiel.

\section{Définition des actions grises}

La veille est partie intégrante du processus d'actions grises. Bien qu'elle n'en constitue qu'un élément, elle joue un rôle capital. Pour Baumard (1997), la veille n'est qu'un outil au service de la stratégie, toutefois la réussite d'un système de veille ne peut y être que difficilement attribué. En effet, l'apport de l'information se conjugue avec des contextes et des pratiques organisationnelles qui débouchent sur la réussite d'opérations. L'intérêt est alors d'étudier l'intégralité du processus partant de la collecte de l'information pour finir par une action décisive.

Afin de définir le concept d'actions grises, nous reprendrons l'échiquier inspiré largement par Usunier et Verna (1994). Traditionnellement, les praticiens positionnent les actions stratégiques relatives à la sphère de l'intelligence concurrentielle sur l'échiquier représenté par la figure 1.

\section{FIGURE 1}

\section{Le positionnement des actions grises}

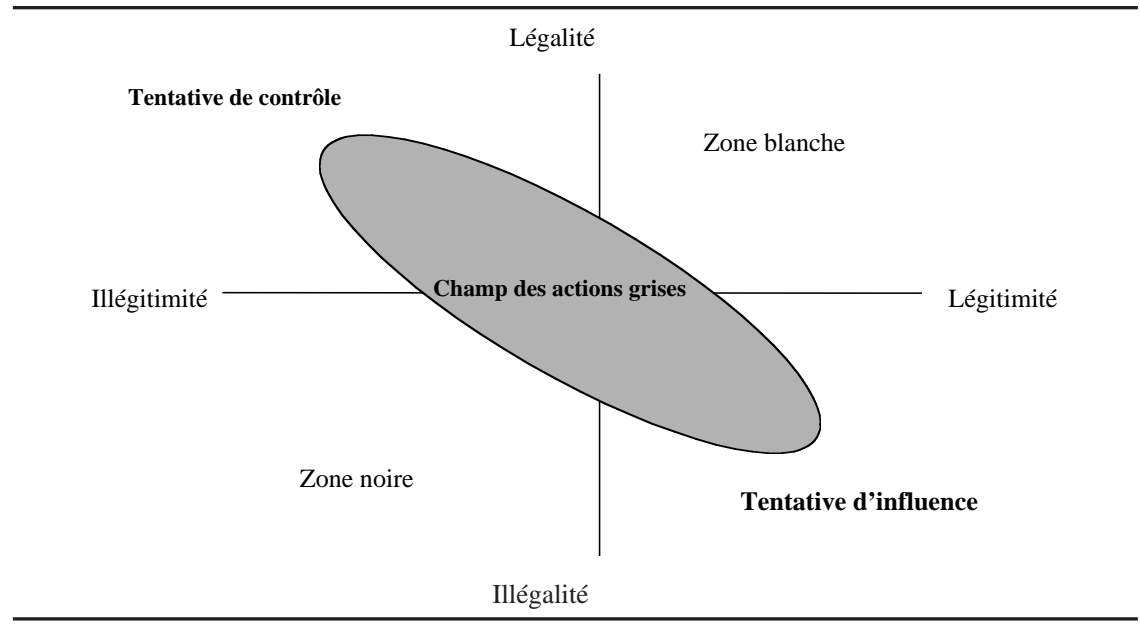

1. Nous nous appuyons sur la grille d'analyse proposée par Tywoniak (1998); celle-ci est développée plus avant. 
Le positionnement des stratégies se définit par rapport à deux axes correspondant au niveau de légalité et au niveau de légitimité.

Le niveau de légalité se définit tout naturellement en rapport à la loi et à son application.

Le niveau de légitimité correspond à un ensemble de coups jugés acceptables sur le plan sociétal, toutes sphères confondues (politique, économique, sociale, juridique). Cet ensemble représente donc ce qui n'est pas strictement juridique.

Ainsi, la pratique des pots-de-vin concernant l'obtention d'un marché étranger est illégale. Mais, du point de vue des intérêts économiques nationaux, la pratique peut paraître légitime. Sur ce plan, le domaine des actions grises constitue un spectre dont les frontières se situent principalement entre volonté de contrôler l'information et désir d'influencer les acteurs. Elles permettent in fine de structurer le jeu informationnel à son avantage afin de configurer les règles en sa faveur. Les actions grises correspondent alors à une compétence distinctive (déterminant interne) fondant un avantage concurrentiel. En résumé, les actions grises visent à atténuer les conditions implicites, théoriques et légales du jeu de la concurrence afin d'obtenir un avantage concurrentiel. Cette définition suggère une progressivité dans le classement de la nature des actions.

La zone grise débute par le non-respect des règles implicites du jeu du secteur. Par exemple, un des acteurs tente de s'approprier la part léonine de la valeur ajoutée du secteur soit par sa position, soit en changeant les règles. Selon Martinet (1998), «ce comportement déviant, souvent qualifié d'hostile par les firmes qui bénéficiaient de la situation antérieure, peut d'ailleurs être le fait d'un concurrent nouveau dans le secteur qui se voit facilement attribuer les traits du voyou ». La zone grise s'installe avec cette transgression de l'ensemble des coups stratégiques permis.

À l'opposé du continuum se situent les actions les plus sombres qui tombent avec certitude sous le coup de la loi. Ces actions se limitent de moins en moins à des pots-de-vin pour prendre des traits de plus en plus complexes.

Le modèle des ressources et des compétences propose un cadre d'analyse à partir duquel nous pourrons étudier la dynamique des actions grises et définir les conditions de l'avantage concurrentiel.

\section{L'avantage concurrentiel dans le modèle des ressources et des compétences (MRC)}

L'émergence des notions de ressource et de compétence en stratégie provient de questionnements liés aux problèmes de diversification (Wernerfelt, 1984). Ainsi, la compréhension de l'avantage concurrentiel repose traditionnellement sur la notion de portefeuille d'activités de l'entreprise. En réalité, ce portefeuille résulte 
de la mise en œuvre des ressources dont dispose la firme. Nous proposons donc de les identifier dans le cas des actions grises et de voir dans quelle mesure elles fondent l'avantage concurrentiel.

Les ressources pour Barney (1991, p. 101) correspondent aux « actifs, capacités, processus organisationnels, attributs de la firme, informations, savoir, etc., contrôlés par une firme qui lui permettent de concevoir et de mettre en œuvre des stratégies susceptibles d'accroître son efficacité et son efficience ». Cette définition permet de cerner la notion mais reste assez floue quant aux différences entre les ressources, au sens large du terme. Ainsi, différents niveaux de ressources peuvent être distingués (Teece, Pisano et Shuen, 1997).

Durand (2000, p. 86) propose la définition suivante : «l'entreprise mobilise des actifs et des ressources auxquels elle a accès et les combine au service de son offre et de ses clients, en faisant appel à des connaissances et à des processus organisationnels qui lui sont propres. Certaines des compétences sont suffisamment spécifiques pour être considérées comme clés, c'est-à-dire qu'elles allient des ressources et des savoir-faire sous une forme et d'une façon telles qu'elles constituent un avantage concurrentiel significatif et durable face aux concurrents ». Le rôle de la stratégie d'entreprise est alors, en référence à ces concepts, de repérer les ressources rares, de les protéger, de les exploiter et d'en créer de nouvelles. Également, le but de la stratégie consiste à les combiner de sorte qu'elles permettent la constitution de compétences spécifiques. Ces dernières fondent alors l'avantage concurrentiel. Celui-ci correspond aux facteurs qui lui permettent d'être plus profitable que ses concurrents et d'obtenir une position concurrentielle forte sur son secteur d'activité (Arrègle et Quélin, 2001). Néanmoins, cet avantage, pour être durable ou soutenable, est lié à l'acquisition de compétences distinctives et peu imitables. Il convient alors de définir des critères permettant de révéler la dimension stratégique des ressources et compétences.

Selon Tywoniak (1998, p. 174-175), dans le MRC «l'avantage concurrentiel ne réside plus nécessairement dans l'exploitation d'une position dominante et protégée sur un marché (ou une niche), mais dans la valorisation supérieure de ses ressources ». Selon le même auteur, pour qu'une ressource ou une compétence apporte un avantage concurrentiel à la firme, il existe six conditions :

Valeur. La ressource ou compétence doit avoir de la valeur pour la firme, parce qu'elle permet de tirer parti d'opportunités de marché ou de neutraliser une menace de l'environnement (Barney, 1991), ou bien parce que cette ressource est supérieure à celles des concurrents et permet donc l'exploitation potentielle d'une rente (Collis et Montgomery, 1995).

Rareté. La ressource doit être rare, c'est-à-dire qu'un nombre limité seulement de firmes peuvent y avoir accès, idéalement une seule (Barney, 1991). 
Non-imitation. La ressource ou la compétence doit être difficilement imitable afin d'empêcher les concurrents de répliquer la stratégie.

Longévité. La ressource peut-elle maintenir l'avantage concurrentiel de la firme dans la durée (Collis et Montgomery, 1995)?

Non-substitution. Pour conserver sa valeur, la ressource ne doit pas avoir de substituts aisément accessibles (Barney, 1991; Collis et Montgomery, 1995).

Appropriation. Selon Barney (1995), afin d'établir un avantage concurrentiel, la firme doit organiser ses processus et sa structure afin de réaliser la valeur potentielle de ses ressources et compétences cardinales.

Ce n'est que lorsque ces six conditions sont respectées qu'une ressource ou une compétence peut permettre d'obtenir un avantage concurrentiel.

Pour le MRC, la stratégie de développement s'articule autour du métier. En effet, les diverses activités de la firme reposent sur l'exploitation conjointe d'un nombre limité de ressources et de compétences cardinales. En outre, une compétence n'est qu'un avantage potentiel. La traduction de la compétence en avantage passe par son incorporation dans l'activité de la firme: de compétence, elle doit devenir compétence cardinale, c'est-à-dire qu'elle doit se conformer aux critères de l'avantage concurrentiel. L'intelligence économique et, plus spécifiquement, les actions grises constituent-elles une compétence susceptible de procurer à la firme un avantage concurrentiel soutenable? Nous testerons dans ce qui suit l'assimilation possible des actions grises à une compétence cardinale. En filigrane, la question qui se pose est la suivante: ces actions représentent-elles plus pour l'entreprise qu'une fonction ordinaire mais nécessaire en interne dont le rôle consisterait à relier les différentes ressources et compétences pour l'action ou un réel moyen d'agir sur l'environnement pour le structurer à son avantage?

\section{Les actions grises: une compétence cardinale?}

Repérer une compétence cardinale en tant que telle n'est pas un exercice aisé lorsqu'il s'agit d'actifs intangibles. Difficilement imitables puisque difficiles à identifier, ceux-ci se trouvent fréquemment être à la source de l'avantage concurrentiel.

Hall (1993) relève quatre types de ressources intangibles. Les premières se matérialisent par des compétences fonctionnelles, les deuxièmes, par des compétences culturelles, les troisièmes, par des ressources positionnelles et les dernières, par des ressources réglementaires.

Partant, Tywoniak (1998) élabore un tableau récapitulatif reproduit dans le tableau 1. 
TABLEAU 1

Intangibles et avantage concurrentiel

\begin{tabular}{|c|c|c|c|c|c|}
\hline \multirow[b]{2}{*}{ Avantage } & \multirow[b]{2}{*}{ Fonctionnel } & \multicolumn{3}{|c|}{ Ressources et compétences } & \\
\hline & & Culturel & Positionnel & Réglementaire & \\
\hline \multirow[t]{2}{*}{$\begin{array}{l}\text { Facteurs } \\
\text { humains }\end{array}$} & $\begin{array}{l}\text { Savoir-faire } \\
\text { des employés, } \\
\text { fournisseurs et } \\
\text { distributeurs. }\end{array}$ & $\begin{array}{l}\text { Perception de la } \\
\text { qualité, aptitude } \\
\text { pour apprendre, } \\
\text { etc. }\end{array}$ & & & Savoir-faire \\
\hline & & & $\begin{array}{l}\text { Réputation, } \\
\text { réseaux. }\end{array}$ & & Actifs \\
\hline $\begin{array}{l}\text { Facteurs } \\
\text { non humains }\end{array}$ & & & $\begin{array}{l}\text { Bases } \\
\text { de données }\end{array}$ & $\begin{array}{l}\text { Contrats, } \\
\text { licences, secrets } \\
\text { commerciaux, } \\
\text { droits de } \\
\text { propriété } \\
\text { intellectuelle. }\end{array}$ & \\
\hline
\end{tabular}

Source : Extrait de Tywoniak (1998) à partir de Hall, 1993, p. 611.

Évaluer avec ferme conviction les ressources intangibles sur lesquelles va reposer l'avantage concurrentiel conféré par les actions grises est une tâche ardue, car il s'agit d'une combinaison de ressources et de compétences (Baumard, 1997). En effet, la réussite des actions grises repose parfois sur le savoir-faire en matière de captation de l'information et de son utilisation pour l'action mais peut reposer sur d'autres avantages également. Si l'entreprise se base sur des compétences fonctionnelles essentiellement, l'aptitude positionnelle est cruciale. Par ailleurs, le service de veille n'est pas toujours la clé de réussite des actions grises. En ce qui concerne les compétences culturelles, les actions grises nécessitent indéniablement certaines aptitudes organisationnelles. La difficulté à constituer une culture adéquate de partage de l'information en est une première illustration (Grandval et Soparnot, 2002). L'entreprise qui maîtrise cet aspect concernant les actions grises obtient indubitablement un avantage concurrentiel, ainsi qu'en témoignent les écrits (Baumard, 1991, 1996 ; Lesca, 1994 ; Martinet et Marti, 1995). L'intelligence économique est l'affaire de tous au sein de l'organisation pour que l'articulation entre l'information et l'action soit efficace.

Lorsque Messier, PDG de Vivendi, proclama hors la loi le pot-de-vin à maintes reprises et à toutes occasions, c'est parce que la pratique est profondément ancrée dans la culture de l'entreprise : «en 1996, Jean-Marie Messier ${ }^{2}$ monte au créneau dans l'éditorial d'une publication destinée aux cadres du groupe : "entre

2. P. Chabert, «Corruption : la lutte pas à pas », Les Échos, 15 février 1999.

Revue internationale P.M.E., vol. 16, nos 3-4, 2003 
un acte illégal et la perte d'un marché, le choix du groupe, c'est la perte du marché. Quiconque déroge à cette règle n'a pas sa place dans le groupe", assène-t-il. Cette ligne rappelée à chaque occasion - discours officiel ou interview - est devenue au fil des ans un leitmotiv». Il en va de même pour son concurrent Suez Lyonnaise qui dispose d'une charte interne de bonne conduite, «les points couverts par la charte sont carrément inclus dans le contrat de travail du cadre. La ligne rouge est désormais clairement tracée. Celui qui la franchit sait qu'il sera licencié ». Toutefois, il ne semble pas formellement que ce soit sur cet aspect que se fasse la différence mais plutôt sur les ressources positionnelles. En effet, la capacité à créer des réseaux d'information, des relations de confiance et des services indirects est capitale, ainsi que la capacité à lier les jeux et à gérer l'opacité (Cova et Salle, 1999).

In fine se pose la question de savoir si ces compétences ou ressources sont à l'origine de l'avantage concurrentiel. Pour ce faire, nous passerons en revue les six conditions que nous confronterons à nos cas. La mesure de l'efficacité des ressources peut poser problème, c'est d'ailleurs là une faiblesse de la théorie. Seule estimation possible, l'évaluation qualitative est retenue (Azzone, Bertelè et Rangone, 1995), nous avons sélectionné des cas où l'impact des actions grises était décisif par rapport aux autres facteurs.

\section{La méthodologie de recherche}

L'impact décisif de ces actions a été le critère déterminant du choix des cas et exemples, pour plus de détails se référer à Grandval (2000). Notre travail s'appuie sur cinq études de cas réalisées entre 1998 et 2000 auprès de PME (actualisation du contexte en septembre 2001). Les noms ont été modifiés pour des raisons de confidentialité évidente. Ces cas ont fait l'objet d'entretiens approfondis non directifs.

Une approche hypothético-inductive a été retenue pour cette recherche. Elle s'inscrit dans un dépassement du clivage entre positivisme et constructivisme, «Il faut dépasser l'opposition entre positivisme et constructivisme, dissiper certaines confusions, par exemple celles résultant d'associations trompeuses entre positivisme et méthodes quantitatives ou, de manière symétrique, entre constructivisme et méthodes qualitatives, pour explorer les différentes implications d'une conception constructiviste en sciences de gestion » (David, 2000, p. 84).

La recherche s'interroge sur la portée stratégique de la maîtrise des actions grises dans des cas de performance vérifiable. Constitue-t-elle une compétence cardinale ? En quoi permet-elle d'obtenir un avantage sur la concurrence?

La présente recherche consiste dans un premier temps à poser un cadre théorique stable (modèles des ressources et des compétences) afin d'élaborer nos propositions et à les confronter à la réalité empirique. Le terrain est toujours susceptible 
de mener à des découvertes heureuses (Miles et Huberman, 1991). Un retour à la théorie, à partir des critères de l'avantage concurrentiel établis par Tywoniak (1998), permet de conclure sur le caractère distinctif de la compétence.

L'identification des actions grises suppose de repérer l'étroite interaction entre les activités et les ressources de l'organisation et celles des acteurs extérieurs. Une telle conception nécessite d'accorder une place privilégiée aux perceptions des dirigeants d'entreprise, à la manière dont ils construisent leur environnement. Il s'agit d'étudier la structuration du jeu que peut susciter la firme. L'approche se fonde sur une analyse descriptive et explicative de la manière dont le dirigeant organise le processus d'information notamment avec les acteurs extérieurs.

Compte tenu des caractéristiques du problème de recherche, une analyse qualitative fondée sur des entretiens en profondeur a été retenue pour analyser de manière empirique des structurations de jeu. Il s'agit de faire émerger des observations, une structure capable de représenter et d'expliquer la réalité (Wacheux, 1993).

\subsection{La méthode des cas}

La démarche se fonde sur l'analyse approfondie de cinq cas. Selon Wacheux (1993, p. 88), l'étude de cas «se définit comme une analyse spatiale et temporelle d'un phénomène complexe par les conditions, les événements, les acteurs et les implications ». Ce choix se justifie par la nécessité d'accéder à des données «enfouies » dans les secrets de l'entreprise, ce qui est particulièrement le cas pour les actions grises par nature censées être protégées de l'observation extérieure. L'étude de cas est pour Yin (1994) une enquête empirique qui étudie un phénomène contemporain dans son contexte actuel, où les limites entre le contexte et le phénomène ne sont pas facilement identifiables et où de multiples sources d'explication sont utilisées. Il était impératif de procéder à une «immersion» dans le réel pour en saisir toute la profondeur et bénéficier de sources de compréhension multiples. La réplication des cas permet en outre d'obtenir une validité externe.

\subsection{La sélection des cas}

Compte tenu des objectifs de notre étude, nous nous sommes centrés sur quelques cas d'entreprises ou, plus exactement, de secteurs pratiquant les actions grises. À la base, l'étude réalisée pour le compte de la chambre de commerce et d'industrie (CCI) de Caen (Alliaume, Condor et Grandval, 1998) nous a fourni une aide précieuse. L'étude CCI fait figure d'étude exploratoire et nous a permis de repérer les entreprises devenues «expertes » en gestion des actions grises. Ensuite, le caractère décisif de l'impact des actions grises a prévalu dans notre sélection des cas. Deux critères majeurs de sélection ont été retenus en cohérence avec les objectifs 
de notre étude. Un premier critère concerne la lisibilité des actions grises et la possibilité d'établir leur impact. Nous avons choisi des cas de moyennes entreprises (moins de 200 salariés) pour la facilité de la démonstration. Ce type d'entreprises repose sur la forte personnalité du dirigeant, sur des comportements peu formalisés, sur des activités généralement spécialisées. Le second critère est la possibilité d'évaluer l'impact des actions grises. L'impact doit être suffisamment identifiable et attribuable aux actions grises.

\subsection{La collecte et le traitement des données}

La démarche qualitative nous a semblé plus à même de saisir l'informel, le non-dit que des questionnaires ne sauraient appréhender. Au total, 15 entretiens pour chacun des cas ont été réalisés avec des dirigeants et leurs collaborateurs immédiats. Deux interviews de deux heures environ avec des dirigeants de chaque entreprise et des dirigeants des entreprises concurrentes. Également, nous avons eu un à deux entretiens (voire plus) d'une durée de deux heures environ avec des collaborateurs proches, ainsi que des acteurs institutionnels (syndicats professionnels, CCI). La réitération des entretiens est indispensable pour avoir accès à une certaine confidentialité. Par ailleurs, le contexte peut être changeant et influer sur les perceptions. Ainsi, en septembre 2001, les acteurs étudiés lors de l'étude initiale (Grandval, 2000) ont été contactés par entretien téléphonique afin de percevoir les évolutions contextuelles.

La collecte des données approfondies fut peu aisée, il a parfois fallu procéder à un véritable jeu de «cache-cache » afin d'accéder aux informations qui traitaient du fond du problème. Il existe en effet de multiples niveaux d'accès à l'information. En conséquence, avec une même personne plusieurs entretiens sont nécessaires pour avoir accès aux données stratégiques. La confiance se gagne progressivement et elle seule permet que s'ouvre la porte du secret, celle qui donne accès à un niveau supérieur de confidentialité. Dans la transmission de l'information, elle joue un rôle clé.

Les rencontres avec les dirigeants reposaient sur un entretien semi-directif. Il s'agissait de retracer les caractéristiques descriptives et explicatives de la structuration du jeu. Pour chaque cas, cela consistait à comprendre la représentation que le dirigeant avait de son entreprise et de son environnement, son positionnement par rapport à cet environnement et, enfin, ses perspectives sur le développement de ses affaires. L'entretien s'articule autour de cinq thèmes.

Un premier thème concerne les caractéristiques descriptives de l'entreprise : l'activité, la nature de l'organisation, la manière dont sont réparties les responsabilités entre les membres de l'entreprise, les changements les plus significatifs intervenus dans les cinq dernières années. Mais il s'agit également de comprendre 
la représentation que le dirigeant se fait de son entreprise par des questions portant sur son parcours personnel et son engagement dans des réseaux professionnels et extra-professionnels.

Un deuxième thème concernait les éléments du tableau de bord en contrôle de gestion, à savoir les coûts, la trésorerie et la valeur ajoutée. Dans un souci de mesurer la performance, nous avons essayé de fixer des ordres de grandeur pour les entreprises afin de tenter de préciser nos conclusions en matière de réussite des actions grises. Toutefois, l'attribution d'une réussite ou d'un échec aux actions grises ne saurait dépendre de ces indicateurs de tableau de bord. Ils ont été recueillis dans le souci de conforter ou d'appuyer nos conclusions.

Le troisième thème porte plus particulièrement sur la perception de l'environnement par le dirigeant. Les relations sont étudiées à la fois du côté de la transaction (fournisseurs, fournisseurs / concurrents, clients) mais aussi de l'interaction (compétiteurs, complémenteurs ; Nalebuff et Brandenburger, 1996). Concrètement, il est demandé aux dirigeants de décrire la perception qu'ils ont des concurrents, de leur stratégie, de leur réseau d'information, de leurs relations avec les autres acteurs à la fois externes et internes au secteur.

Le quatrième thème porte plus particulièrement sur le système de renseignement en tant que compétence organisationnelle. Il s'agit d'établir comment se fait l'articulation entre l'information et l'action.

Le cinquième thème tente de faire la synthèse des thèmes précédents. Les premiers points abordés portent essentiellement sur les éléments et les relations qui, selon le dirigeant, ont entraîné la structuration du jeu, faisant appel à la perception et à la représentation qu'il se fait du jeu et des joueurs. Le second point concerne plus particulièrement les acteurs non cités par le dirigeant et qui ont pu jouer un rôle. Il s'agit de tester si le dirigeant a une connaissance des acteurs extérieurs situés dans son environnement et, le cas échéant, de connaître les raisons pour lesquelles il n'a pas établi de lien avec eux. Enfin, l'entretien se termine par une question à coloration prospective sur la façon dont le dirigeant envisage l'évolution du secteur, de son métier dans les cinq ans à venir et quelle stratégie il souhaite mettre en œuvre.

Les données recueillies ont été traitées selon la méthode préconisée par Miles et Huberman (1991). Notre compréhension approfondie des contextes a permis d'orienter nos interprétations. À partir de ces «intuitions », nous avons construit notre grille de codage théorique des données recueillies. Les codes retenus mettent en évidence l'impact décisif des actions grises. Le retour à la théorie permet, après avoir démontré le caractère décisif de la maîtrise des actions grises, de soumettre cette compétence aux critères de l'avantage concurrentiel. 


\subsection{Présentation des cas}

Les cas étudiés visent à refléter non pas une pratique en particulier mais plutôt une dynamique d'organisation des actions grises qui tient compte des interdépendances. Ils représentent des exemples de réussite que nous pouvons attribuer sans beaucoup de doutes aux actions grises. Nous nous attacherons dans cette partie à présenter les cas en axant notre analyse sur la nature des politiques en matière d'actions grises.

\subsubsection{Cas $D B N$}

DBN a acquis une position centrale au sein d'un réseau, ce qui lui permet de structurer le jeu à son avantage grâce à son aptitude à capter l'information pertinente. DBN est un sous-traitant automobile. Sur ce marché, le degré d'intégration de la production varie selon les types de pièces à fabriquer. Certains fournisseurs traitent le produit plus en aval ou en amont que d'autres. De même, quelques fournisseurs ont une meilleure maîtrise de certains processus de production. Ce faisant, les acteurs sont amenés à collaborer pour obtenir des contrats difficiles. En effet, les constructeurs automobiles sont de plus en plus exigeants en termes de qualité, de productivité et donc de coûts. Une constellation d'entreprises gravite autour d'une firme pivot, également appelée sous-traitant de premier rang.

DBN a acquis le statut de fournisseur de premier rang à la faveur d'une politique de réduction du nombre de fournisseurs instaurée par les constructeurs. La firme pivot s'est appuyée sur ce mouvement pour s'imposer comme chef de file. Son rôle devient alors d'organiser le secteur afin de pouvoir répondre aux commandes et exigences des constructeurs. À la base, la collaboration qui s'est édifiée s'effectuait uniquement pour la sous-traitance automobile. Le pouvoir de la firme pivot se concrétise dans la redistribution des contrats, dont il a la charge au sein du groupe. Conséquence: les concurrents du secteur sont contraints de négocier avec la firme pivot et non plus avec les constructeurs. Les concurrents se sont trouvés coupés des relations et informations avec le constructeur. La firme pivot a donc indirectement obtenu le pouvoir de filtrer, transformer et redistribuer l'information selon sa convenance.

D'après les acteurs du secteur, cette coupure d'avec le constructeur les a placés dans l'incertitude avec une dépendance informationnelle à l'égard de la firme pivot. De ce fait, la négociation sur le partage de la valeur ajoutée est fortement biaisée par l'asymétrie d'information.

Dans le cas DBN, le dirigeant de la firme pivot joue le rôle de conseiller pour les autres entreprises. Toutefois, ce personnage clé est partie prenante donc il a tendance à orienter ses conseils pour servir la stratégie de sa propre firme; il dispose d'une certaine capacité à structurer le jeu à son avantage. Nous pouvons 
parler d'intégration informationnelle. En effet, la firme pivot contrôle les informations stratégiques et redistribue l'information conformément à ses intérêts. Si les relations sont parfois économiques, elles sont surtout informationnelles (savoirfaire, évolution du marché, possibilité d'organiser le marché, etc.). Les autres membres de la structure ont intérêt à demeurer en son sein. En effet, leur appartenance au réseau leur apporte une économie sur la recherche et le traitement de l'information qui est considéré de bonne qualité eu égard à l'expérience et aux liens qu'a su créer le dirigeant de la firme pivot. S'extraire du réseau, c'est se couper d'occasions de coopération, de partages d'expériences et d'informations sur le marché.

Le pouvoir informationnel de la firme pivot ne se limite pas à l'interface constructeurs et membres du groupe. Le dirigeant a enrichi ses sources d'information. Il a établi des contacts privilégiés avec les organismes consulaires et territoriaux. Par ces liens, il a pu accéder (ou fait réaliser) à des études nationales et internationales sur le secteur auprès de ces organismes. Ainsi, il s'est assuré une veille stratégique efficace et large, le coût étant supporté par ces organismes.

En ce qui concerne l'information informelle, il a accès notamment à toute l'information locale. Représentant du secteur, il a l'occasion de participer à nombre de foires et salons. Le dirigeant acquiert une parfaite connaissance du secteur. Toutefois, s'il a réussi à transférer une partie du coût monétaire de sa veille, le coût en termes de temps demeure considérable (la moitié de ses heures travaillées). Les acteurs du secteur ont bien entendu conscience du pouvoir qu'il a acquis en termes d'information. Toutefois, eu égard au coût et à l'utilisation qu'ils en ont, ils préfèrent laisser ce rôle au dirigeant de la firme pivot. Les concurrents ont estimé ne pouvoir avoir accès au même niveau d'information.

\subsubsection{Cas «Les Tilleuls»}

Cette entreprise a développé une stratégie de "camouflage» afin de créer un brouillard autour de sa stratégie de filialisation. Il s'agit d'apparaître comme un «artisan» soucieux de la qualité des prestations et du confort de ses «clients» et non comme un groupe financièrement intégré.

Ce groupe de maisons de retraite (MDR) comprend quatre maisons de retraite d'une capacité moyenne de 120 lits. En comptes consolidés, il pourrait prétendre atteindre le top 20 du classement des MDR en termes de taille. En effet, le marché de l'hébergement privé commercial pour personnes âgées est très éclaté. Le dirigeant tenait à ce que ses maisons de retraite apparaissent comme des établissements indépendants alors qu'existent pour ces établissements une centralisation de l'administratif et un partage du personnel (animateurs, infirmières, kinésithérapeutes, médecins). Il s'agissait d'un groupe de fait puisque les détenteurs des capitaux sont identiques et dans les mêmes proportions pour chaque établissement. 
L'hébergement pour personnes âgées est un sujet sensible à connotation éthique forte. En effet, d'après l'ensemble de nos entretiens réalisés auprès des directeurs de MDR et des autres acteurs du secteur, la plupart des familles sont scrupuleuses à l'idée de placer leurs aïeux en MDR, d'autant plus que les récents scandales sur les MDR non conformes aux règles de sécurité ont renforcé les craintes à l'égard de la qualité des prestations des MDR. Dans un tel contexte, l'hébergement privé commercial acquiert la réputation de «faire de l'argent sur le dos des personnes âgées », selon les propos d'une directrice d'une MDR. Cette perception par les familles des patients est confirmée par l'ensemble des acteurs du secteur. Dès lors, les groupes de MDR partent avec un déficit d'image, surtout lorsque ceux-ci utilisent des méthodes de démarchage auprès des prescripteurs que sont les médecins. Les médecins n'appréciaient pas toujours ces méthodes actives. In fine, les groupes ont acquis une image détériorée. En conséquence, la stratégie du groupe «Les Tilleuls» a été de dissimuler la dimension du groupe pour mettre en avant l'indépendance des établissements. Tout ce qui a trait au groupe est soigneusement masqué. L'image d'un établissement à taille humaine, haut de gamme, constitue un avantage concurrentiel. Le choix marketing du directeur est d'insister sur la qualité du service à la personne âgée dans chaque établissement. La révélation de l'appartenance à un groupe aurait, pour ce cas précis, un effet négatif sur l'image alors que, dans la plupart des autres secteurs, l'effet est bien souvent inverse.

\subsubsection{Cas Indep}

Le cas traite de sept MDR indépendantes regroupées en association, Indep. Elles mènent une politique d'influence afin de structurer le jeu à leur avantage et «d'éliminer» les MDR ne respectant pas certaines «normes » de qualité. L'objectif du groupe des sept MDR a été de se réunir afin d'amorcer une démarche qualité qui permettra de rendre visible la qualité de leurs prestations. Le but ultime est de leur donner force obligatoire en proposant aux instances européennes de légiférer sur des conditions minimales de normes. C'est un moyen affiché d'éliminer les «moutons noirs » de la profession qui ne pourront s'aligner sur ces normes. Avec l'amélioration de la qualité, les MDR espèrent faire augmenter la valeur perçue de leur prestation et in fine leur valeur ajoutée. Cela signifie-t-il que les patients seront disposés à payer davantage que ce qu'ils paient à ce jour? Il semblerait que non. Le prix de l'hébergement et des soins en MDR est si élevé qu'une aide est fréquemment indispensable. Le prix tend d'ailleurs à augmenter du fait de l'entrée de plus en plus tardive en MDR de patients de moins en moins valides, ce qui entraîne de ce fait des coûts croissants. L'enjeu est donc de convaincre l'État, les organismes sociaux et les collectivités locales d'accorder une aide la plus large possible, couvrant notamment les coûts les plus élevés, c'est-à-dire les soins. La réalisation de l'objectif passe par une contrepartie: il s'agit pour les membres 
d'Indep d'être crédibles en tant qu'établissement de soins, ce qui implique l'acceptation de contrôles, de normes strictes mais également un assainissement du secteur. Les financeurs ne sauraient soutenir des établissements aux prestations douteuses. Les préoccupations sur la qualité sont un signal envoyé tant aux patients potentiels qu'aux financeurs sur leur engagement ferme à clarifier la qualité des prestations du secteur. C'est un moyen d'écarter des concurrents qui, s'ils présentent un prix de journée faible, offrent de piètres prestations conduisant sporadiquement à des scandales.

\subsubsection{Cas Santix}

Afin d'assurer sa politique de croissance, la société Santix a mis en place des manœuvres indirectes pour acquérir des établissements supplémentaires à moindre coût.

Santix gère et détient plusieurs cliniques privées. Cette entreprise est filiale d'un grand groupe: Groupix, qui possède plusieurs filiales à l'œuvre dans différents métiers. Santix dispose d'une politique de groupe et d'une collaboration entre les filiales pour servir les finalités de chacune d'entre elles. Ainsi, le groupe Santix, dans une politique d'expansion, souhaitait acquérir des établissements. L'entreprise est informée en amont des projets d'extension, de rénovation ou de construction neuve par une autre filiale appartenant au secteur du bâtiment et des travaux publics (BTP). Cette dernière est en contact avec les architectes qui travaillent fréquemment pour elle. Les architectes se trouvent être en amont des projets, ce qui est idéal pour le repérage des chantiers à venir.

Ainsi prévenu du projet de construction d'un actif entièrement nouveau par la clinique Clinix, établissement indépendant, une politique visant à mettre en difficulté l'établissement et le contraindre à la cession a alors été mise en œuvre. Dans la présentation qui suit, les chiffres présentés sont, pour des raisons de confidentialité, quelque peu transformés. En revanche, les proportions, elles, sont conservées.

En 1995-1996, au vu des nouvelles normes de classement des cliniques et des directives concernant les renouvellements d'autorisation d'exploitation de lits de courts séjours, Clinix décide d'entreprendre une restructuration. Les objectifs consistent en la mise aux normes, l'amélioration du classement et l'obtention d'un tarif dérogatoire. Ce dernier objectif avait été soutenu par leurs consultants qui avaient monté avec succès des dossiers de dérogations tarifaires dans d'autres régions (Nord-Pas-de-Calais), dans lesquelles le prix de journée est largement supérieur à celui de la région de Clinix. Cette dérogation permettait de rembourser le financement de l'opération (3,21 millions d'euros) avec une marge de sécurité satisfaisante, étant entendu qu'ils ne disposaient d'aucun autofinancement au départ. 
Au cours du chantier, après les visites des commissions de sécurité, un certain nombre de travaux supplémentaires furent imposés ( 0,84 million d'euros), s'ajoutèrent des dépassements non prévus ( 0,76 millions de francs). Au total, une dépense supplémentaire d'environ cinquante pour cent.

L'architecte parisien se révéla de mèche avec Santix dans l'organisation d'un dépassement d'enveloppe de travaux afin de mettre la clinique en difficulté et de lui proposer une participation dans son capital. En effet, un architecte de métier, habitué aux travaux de ce type, pouvait-il ignorer les normes et ne pas prévoir les dépassements ? En outre, l'architecte a influencé le choix du constructeur, à savoir la filiale BTP de Groupix. En revanche, nous n'avons pu déterminer clairement si les consultants, par leur anticipation d'un régime dérogatoire optimiste, avaient aussi collaboré avec Santix.

Par ailleurs, lors de la visite de conformité et de classement, la caisse régionale d'assurance maladie (CRAM) mit en réserve sa décision quant au tarif dérogatoire eu égard aux difficultés financières de l'établissement. La décision de mise en cessation de paiement fut prise deux ans plus tard. Un an encore après, le tribunal décide de la reprise par Santix. Les conditions accordées au repreneur sont un étalement des dettes accepté par les banques et les fournisseurs, une restructuration du personnel achevée, en contrepartie d'une recapitalisation par Santix. Au final, la reprise par Santix devrait lui coûter (rachat plus recapitalisation) une quinzaine de millions de francs. Il s'agit d'un montant avantageux pour un actif récent, de bonne qualité. Par ailleurs, l'étalement de la dette et un personnel restructuré créent les conditions d'une pérennité et d'une rentabilité pour l'établissement.

L'ARH (la décision ne dépendant plus de la CRAM) attendait l'avis du tribunal concernant la dérogation tarifaire. Pour l'heure, nous ne connaissons pas sa décision.

Culturellement, les praticiens, personnes stratégiques pour la qualité des prestations des cliniques, aiment à être indépendants. Traditionnellement, ils sont actionnaires eux-mêmes des établissements. Ils manifestent de la méfiance à l'égard des grands groupes de santé. Leur perception étant qu'une politique de groupe centralisée remettrait en cause leur indépendance, car elle leur imposerait notamment des normes dans l'exercice de leurs actes et un contrôle de gestion. En conséquence, il était difficile pour Santix d'acquérir des établissements en raison de l'opposition des praticiens. Pour contourner le problème, il fallait acculer les cliniques à céder des parts. En effet, le cas relaté plus haut n'est pas un cas isolé, c'est désormais une pratique bien connue et qui a cessé pour cette raison. D'ailleurs, la politique de croissance au niveau de la France ne constitue plus une priorité pour Santix. Dans un premier temps, la pratique ne devait pas être connue. Le lien ne devait pas être fait avec Santix. La manœuvre devait être orchestrée par un élément extérieur au groupe: l'architecte. Celui-ci avait pour rôle de se porter 
candidat sur des projets, soit détectés par lui, soit repérés par Santix grâce à une politique de veille. Ensuite, le choix était fait d'un constructeur (filiale de Groupix) dont le lien avec Santix ne paraissait pas évident. En tout cas, une politique de coordination entre les groupes n'était pas soupçonnable. Le non-dévoilement des pratiques par les différents salariés des entités est notamment assuré par l'engagement de leur réputation (l'architecte) si ce n'est de leur implication pénale.

\subsubsection{Cas Magma}

La stratégie de l'entreprise Magma est de jouer sur les brevets d'invention pour embrouiller la concurrence.

Cette moyenne entreprise, spécialisée dans la sous-traitance automobile, développe des produits d'électronique embarquée. L'innovation technologique joue ainsi un rôle central dans la construction d'un avantage concurrentiel. La veille sur les brevets est une activité courante dans le monde industriel. En effet, le titre, la description et les revendications, par la précision exigée, sont des indications précises de ce que les concurrents peuvent envisager comme produits futurs à partir de ces technologies. Les concurrents peuvent donc commencer à amorcer une riposte bien avant que le nouveau produit ne naisse. En outre, au moment de la demande de brevets, les entreprises ne savent pas toujours avec certitude quelles seront les conséquences de l'invention en termes de produits. Ils ne peuvent donc formuler clairement et de façon exhaustive leurs revendications. Un concurrent plus clairvoyant peut donc sortir des produits non couverts par les revendications.

Afin de pallier ces risques, Magma a adopté une technique d'opacification. Pour ne pas éveiller les soupçons de ses concurrents avec le dépôt du brevet, l'entreprise émet un flux continu de demandes de brevets pas toujours pertinents ou industriellement intéressants. De plus, elle noie ses principales demandes de brevets dans d'autres demandes sans objet pour l'entreprise. Elle consent un effort particulier, dans le même temps, à l'anticipation des répercussions des inventions afin de pouvoir déposer des revendications suffisantes et même au-delà de ce qui est utile. L'entreprise détourne le règlement en s'appuyant sur les faiblesses de celui-ci. Le système des brevets étant trop transparent sur les intentions de la firme déposante, il est nécessaire pour celle-ci d'épaissir le brouillard. L'entreprise, par ailleurs, induit en erreur la concurrence en produisant des titres erronés pour des brevets inintéressants. Ainsi, la nullité de celui-ci peut être prononcée mais ne la gênera pas. En revanche, sachant que la veille de brevets se fait essentiellement sur le titre, les travaux de veille des concurrents s'en trouvent considérablement entravés pour un coût de vérification (financier et temporel) important. 


\section{Les actions grises à l'épreuve des critères de l'avantage concurrentiel}

La démarche consiste à confronter le concept d'action grise avec chaque critère qui fonde l'avantage concurrentiel selon le MRC. Cette mise à l'épreuve s'appuie sur les cas étudiés et permettra de postuler que les actions grises confèrent aux firmes un avantage concurrentiel durable.

\subsection{La valeur}

Les actions grises, lorsqu'elles permettent à l'entreprise d'obtenir un marché, ont incontestablement de la valeur pour la firme. Elles servent plusieurs missions, que ce soit de défense ou d'attaque. Dans le premier cas, il s'agit de «neutraliser une menace de l'environnement». Dans le second cas, il s'agit «de tirer parti d'opportunités de marché ».

Dans le cas DBN, la capacité de la firme pivot à organiser le secteur pour répondre aux commandes du donneur d'ordres est une compétence qui lui permet de tirer parti d'opportunités de marché. Sans cette organisation et la capacité à faire suivre les autres membres du groupe, l'entreprise serait de taille trop faible pour répondre aux exigences des donneurs d'ordres et, en conséquence, serait écartée du marché.

Dans le cas Santix, l'organisation au niveau d'une politique globale de groupe a permis d'acquérir une clinique à moindre coût en acculant les propriétaires à la vente.

Concernant Les Tilleuls, le fait d'apparaître comme établissement unique permet de neutraliser la menace qui est d'apparaître comme une entreprise qui veut faire de l'argent sur le dos des personnes âgées.

Second aspect, le fait pour les membres d'Indep de se réunir pour fixer des normes est un moyen de neutraliser les concurrents « moutons noirs » qui dégradent l'image du secteur.

L'entreprise Magma dépose quantités de brevets afin d'empêcher que la concurrence ne décèle le brevet qui annonce le lancement ou l'amélioration de produit. La capacité à brouiller les cartes est une compétence visant à neutraliser la menace de l'environnement que constitue le système de veille des concurrents.

\subsection{La non-imitation}

La stratégie doit demeurer inimitable au risque de voir disparaître l'avantage concurrentiel. Dans ces conditions, lorsque la règle générale d'un secteur est la distribution d'un pot-de-vin par exemple, il est pertinent de se demander quel élément fait la 
différence, sinon l'emporterait le mieux-disant financier. Dans quelles conditions un acteur l'emporte sur un autre ? La compétence à repérer les bons réseaux d'influence semble constituer un facteur discriminant au vu de nos études de cas. La constatation a une dimension plus générale. À la suite de l'étude sur l'intelligence économique en Basse-Normandie (Alliaume, Condor et Grandval, 1998), il est apparu que l'identification à l'étranger des bons acteurs d'influence afin d'accéder aux marchés en toute quiétude est une demande forte et donc une compétence dont ne disposent pas toutes les entreprises. C'est d'ailleurs une proposition qui a été opérationnalisée et concrétisée par la CCI de Caen à la suite de l'étude.

Par ailleurs, en ce qui concerne les actions grises, les acteurs entretiennent un voile pour qu'à la fois les instances judiciaires et la concurrence ne puissent avoir accès à leur pratique. Auquel cas, l'imitation ou la dénonciation seraient à craindre, détruisant par là même l'avantage reposant sur cette compétence ainsi dévoilée.

La firme pivot du cas DBN s'est assurée que ses compétences ne pouvaient être imitées en se positionnant au centre des flux d'informations. Elle a par ailleurs coupé les relations entre les donneurs d'ordres et les entreprises coopérantes et a monopolisé les relations avec les autres organismes.

Les Tilleuls pratique la discrétion qui vise à ne pas se faire repérer pour ne pas être l'objet de l'attention des concurrents, maintenant ainsi ses compétences au secret. Dans ce cas précis, son action est à caractère défensif afin de protéger les autres compétences qui caractérisent sa politique de préservation d'une image éthique.

Concernant Indep, la non-imitation réside dans la non-possibilité que possèdent les «moutons noirs » à se structurer en groupe pour influer sur les normes. En effet, l'objet de ces mauvais concurrents est d'éviter l'établissement de normes sur lesquelles ils savent ne pouvoir s'aligner en raison de leur choix de gestion qui vise à minimiser les coûts, notamment en personnel, au détriment de la qualité et de l'image.

Cette politique défensive est également le cas de Magma. La compétence à protéger les intentions stratégiques est cardinale. Son absence entraînerait l'anticipation de ses actions par les concurrents qui grèverait les bénéfices attendus du lancement de produits. Toutefois, les brevets déposés en tant que leurre doivent avoir un minimum de crédibilité. Cela implique des compétences au brouillage des intentions pour assurer la crédibilité et la non-révélation des intentions.

Santix dispose assurément d'un avantage non imitable par la définition d'une politique de groupe qui fait appel à une palette de compétences et de métiers. Les concurrents qui n'appartiennent pas à un groupe bénéficiant d'un tel catalogue ne peuvent d'emblée imiter les compétences. En outre, le secret qui entoure ces 
pratiques aux allures quasi illicites implique une faible visibilité pour les concurrents. Le secret est assuré par les multiples décideurs du groupe dont la responsabilité pénale est impliquée en raison de leurs actes.

\subsection{La longévité}

Les actions grises, notamment parce qu'elles exigent des structurations de réseaux complexes, produisent essentiellement leurs effets dans le long terme. Ainsi, la compétence est censée s'affiner dans le temps. Connaissant un effet d'expérience, elle a tendance à renforcer l'avantage concurrentiel avec les années. Toutefois, les mutations dans les secteurs, l'irruption de nouveaux concurrents, l'émergence de nouvelles règles (notamment juridiques) peuvent remettre en cause la longévité. Il s'agit d'ailleurs là de la principale critique adressée au MRC: lorsque les règles du jeu changent, la compétence sur laquelle l'avantage concurrentiel repose peut alors ne plus convenir.

Dans le cas DBN, le dirigeant de la firme pivot a accru son emprise sur le réseau au cours du temps. De ce fait, sa compétence, corrélativement à son hégémonie, s'accroît avec le temps. Toutefois, une trop forte contestation de ce rôle par les autres firmes peut remettre en cause la durabilité de son rôle de leader.

Dans le cas des Tilleuls, si le voile se lève sur la nature réelle de sa dimension, l'image risque de s'en ressentir. Or, le secret, de façon générale, a vocation à être découvert, particulièrement lorsqu'il existe un milieu fortement structuré tel que le milieu médical où l'information circule abondamment. La longévité n'est pas assurée. Toutefois, la réputation acquise vient compenser le risque de dévoilement. En revanche, les groupes concurrents peuvent davantage repérer ce concurrent rentable et tenter de copier ses compétences reconnues par le milieu en matière de soins tant physiques que psychologiques.

Façonner le marché en définissant les normes permet à Indep d'obtenir un avantage concurrentiel durable et visible. La longévité est donc assurée. Le risque vient du non-prolongement du travail entrepris sur les normes et la qualité par effritement de la collaboration. Dans ce cas, la définition de normes obligatoires ne peut être promulguée par les autorités de tutelle.

Concernant le cas Santix, la politique de groupe ne peut être pratiquée par les concurrents qui ne disposent pas d'une intégration au sein d'un grand groupe. La longévité peut être maintenue. Toutefois, en cas de cession de la filiale (ce qu'il advint), l'avantage disparaît. Également, le dévoilement des pratiques obligerait le groupe à ne plus utiliser cette compétence à la limite de la légalité.

Dans le cas Magma, la méthode du leurre utilisée est telle qu'il est difficile pour les concurrents de déterminer quel est le brevet stratégique pour la firme. Toutefois, une méthode d'enquête (y compris l'espionnage industriel) et de 
décryptage peut être employée par les concurrents. La démarche exige néanmoins des moyens considérables que ceux-ci ne sont peut-être pas prêts à engager. De plus, la difficulté, le risque juridique, la lourdeur et la non-certitude d'une telle pratique permettent de croire à la longévité.

\subsection{La rareté}

La compétence à structurer les actions grises dépend de la flexibilité organisationnelle et des aptitudes à l'apprentissage. La capacité tant en interne qu'en externe à mobiliser des hommes filtres constitue une ressource rare, car ces hommes eux-mêmes sont rares. En effet, leur métier est de fournir et d'opérationnaliser l'information stratégique, matière rare également.

Dans le cas DBN, le dirigeant de la firme pivot s'est imposé comme un véritable homme filtre, producteur d'informations pertinentes dont les compétences en tant que telles sont unanimement reconnues, que ce soit par les dirigeants des autres firmes internes au réseau ou bien extérieures au groupe. Comme les autres dirigeants estiment ne pouvoir égaler son niveau de compétence, ils préfèrent lui déléguer ce rôle. Ainsi, la difficulté d'acquisition de cette compétence conduit à l'abandon de cette compétence par les concurrents.

Dans le cas Santix, le groupe est le seul acteur du secteur à pouvoir bénéficier de l'intégration dans un groupe et, plus particulièrement, à pouvoir utiliser la palette de métiers du groupe pour parvenir à ses fins.

Concernant Magma, la rareté de la compétence à brouiller les pistes se trouve dans la complexité à édifier un plan de cryptage. Mais ce n'est pas tant la rareté de la compétence à troubler les données que la conséquence en termes de rareté de la ressource clé (que constitue l'information) qui est source d'avantage concurrentiel. En effet, la pléthore d'informations est provoquée afin d'égarer les concurrents sur ses intentions stratégiques alors que, parallèlement, Magma continue à observer les brevets de ses concurrents. L'avantage concurrentiel provient de la capacité à créer cette asymétrie d'information.

\subsection{La non-substitution}

Dans le jeu des affaires business to business, l'entreprise peut difficilement faire l'économie d'une politique d'intégration dans le milieu. En conséquence, les substituts sont difficiles à construire.

C'est également cette situation qui prime dans le cas DBN. La firme pivot s'est placée au centre du réseau et a su en tirer profit. La coopération entre acteurs implique la circulation de l'information. Nulle compétence ne saurait compenser une absence d'information. Les acteurs hors réseau (les entreprises LJLG et 
REED $^{3}$ ) ont connu des difficultés en se coupant du réseau. La politique de prix bas, pratiquée par leurs soins, n'a pas compensé la non-intégration au sein du groupe.

Dans le cas Santix, des moyens de substitution efficaces n'ont pu être opposés à la politique de rachats du groupe et la constitution d'unions d'établissements indépendants n'a pas constitué un pis-aller. En effet, de tels groupements manquent de cohérence organisationnelle. De plus, ils ne protègent pas nécessairement du rachat par un grand groupe. En revanche, ils sont moins vulnérables à la stratégie de mise en difficulté. En effet, le groupement leur donne les moyens d'accéder à des expertises et à des retours d'expériences de confrères.

Dans le cas des Tilleuls, il n'existe pas d'alternative. La loi du milieu exige un traitement en termes d'image et d'éthique en contrepartie d'une implication financière des autorités de tutelle. Dans le cas d'Indep, la solution la moins coûteuse est de cacher la dimension pour satisfaire aux exigences en termes d'image. Une campagne marketing visant à entretenir une bonne image serait bien plus onéreuse et son résultat, aléatoire.

L'autre partie de l'alternative pour Magma serait de ne jamais breveter ses inventions sur lesquelles sont basés ses nouveaux produits afin de ménager les effets de surprise. Mais la rapidité d'imitation possible par les concurrents rend impérieuse la nécessité d'une politique d'opacification.

\subsection{L'appropriation}

La compétence à structurer les actions grises en appelle à plusieurs compétences cardinales telles que la flexibilité organisationnelle, la compétence à capter, traiter, diffuser l'information, compétence à intégrer le milieu, etc. Dégager la valeur potentielle des ressources et compétences cardinales implique de parvenir à combiner ces compétences pour une articulation efficace entre le but, l'information et l'action. Pour l'ensemble des cas, nous verrons en quoi les buts ont été atteints.

Dans le cas DBN, le résultat de son rôle de firme pivot se manifeste par une valeur ajoutée supérieure (et de loin) à celle dégagée par les autres acteurs du secteur et du réseau, notamment les concurrents directs.

Dans le cas Santix, le résultat s'est concrétisé par l'acquisition de la clinique visée à un prix modeste puisque l'établissement était en redressement judiciaire. De plus, la dimension de la filiale et son appartenance à un grand groupe a pesé dans l'attribution à cette firme de l'établissement par le tribunal de commerce.

3. Ces entreprises sont concurrentes de l'entreprise DBN et n'appartiennent pas au réseau. 
Dans le cas Magma, le dépôt systématique de brevets, même les plus insignifiants (en termes d'exploitation), a ralenti le degré d'anticipation des concurrents.

Dans le cas d'Indep, les résultats pour le groupe ne sont pas clairement identifiables. Toutefois, l'idée de normes européennes fait son chemin. Les établissements les plus douteux sont victimes d'une répression plus sévère, conséquence d'une mise en évidence des différents niveaux de qualité.

Dans le cas des Tilleuls, les journaux spécialisés ne l'identifient pas en tant que groupe, elle est une des rares MDR privées à but lucratif (13\% des MDR selon une enquête de l'UNEPA) à réaliser un résultat supérieur à $10 \%$ du CA, résultat de son positionnement haut de gamme.

\section{Synthèse : la capacité à structurer les actions grises, une compétence cardinale?}

En synthèse des cas présentés précédemment, le tableau 2 tente de mettre en exergue la dynamique des actions grises permettant de les qualifier de compétence cardinale. Le tableau 3 détaille les types d'actifs intangibles sur lesquels repose cette compétence cardinale pour chaque cas.

Le tableau 2 synthétise les réponses qu'ont pu fournir les acteurs par rapport aux six conditions constitutives d'une compétence cardinale à structurer des actions grises.

Dans le cas des actions grises, les conditions se vérifient, dans bien des cas, de façon indirecte ou par combinaison avec d'autres compétences. Également, leur rôle peut être de raréfier des ressources, de là, elles tirent leur caractère cardinal. Le tableau 2 tente de montrer néanmoins en quoi les actions grises peuvent procurer, par leur aspect décisif, un avantage concurrentiel.

Le tableau 3 tente de relever, pour les différents cas, les types d'actifs intangibles qui favorisent principalement la structuration des actions grises au sein des entreprises. Ces actions grises elles-mêmes confèrent l'avantage concurrentiel.

Les actions grises, en tant que compétences internes, n'en sont pas moins tournées vers l'extérieur. En ce sens, leur rôle est d'organiser, pour certaines opérations, la défense et l'attaque vers l'extérieur.

La figure 2 représente le positionnement de chaque cas étudié sur l'échiquier légitimité / légalité. 
TABLEAU 2

Structurer les actions grises : une compétence cardinale?

\begin{tabular}{|c|c|c|c|c|c|}
\hline & DBN & Les Tilleuls & Indep & Santix & Magma \\
\hline Valeur & $\begin{array}{l}\text { Compense la } \\
\text { faiblesse de } \\
\text { la taille. }\end{array}$ & $\begin{array}{l}\text { Positive } \\
\text { l'image. }\end{array}$ & $\begin{array}{l}\text { Neutralise les } \\
\text { moutons noirs. }\end{array}$ & $\begin{array}{l}\text { Accule les } \\
\text { propriétaires à } \\
\text { la vente pour un } \\
\text { faible prix. }\end{array}$ & $\begin{array}{l}\text { Neutralise le } \\
\text { système de } \\
\text { veille des } \\
\text { concurrents. }\end{array}$ \\
\hline Non-imitation & $\begin{array}{l}\text { Au centre des } \\
\text { flux } \\
\text { d'informations. }\end{array}$ & $\begin{array}{l}\text { Voile sur la } \\
\text { position exacte. }\end{array}$ & $\begin{array}{l}\text { La non- } \\
\text { imitation est } \\
\text { la finalité. }\end{array}$ & $\begin{array}{l}\text { Une palette de } \\
\text { compétences et } \\
\text { de métiers que } \\
\text { seul le groupe } \\
\text { peut proposer. }\end{array}$ & $\begin{array}{l}\text { Technique du } \\
\text { leurre complexe } \\
\text { en matière de } \\
\text { brevets. }\end{array}$ \\
\hline Longévité & $\begin{array}{l}\text { Emprise } \\
\text { croissante de } \\
\text { la firme pivot. } \\
\text { Risque de } \\
\text { rébellion. }\end{array}$ & $\begin{array}{l}\text { Secret difficile } \\
\text { à maintenir. }\end{array}$ & $\begin{array}{l}\mathrm{La} \\
\text { collaboration } \\
\text { peut s'effriter. }\end{array}$ & $\begin{array}{l}\text { Intégration dans } \\
\text { un groupe. } \\
\text { Risque de } \\
\text { cession ou de } \\
\text { dévoilement. }\end{array}$ & $\begin{array}{l}\text { Difficulté à } \\
\text { décrypter le } \\
\text { leurre. } \\
\text { Risque de } \\
\text { pratiques noires } \\
\text { des concurrents. }\end{array}$ \\
\hline Rareté & $\begin{array}{l}\text { Accès exclusif } \\
\text { à l'information. } \\
\text { Compétence } \\
\text { au filtrage. }\end{array}$ & Non déterminé & Non déterminé & $\begin{array}{l}\text { Seule firme à } \\
\text { pouvoir } \\
\text { bénéficier de } \\
\text { l'intégration } \\
\text { dans un groupe. }\end{array}$ & $\begin{array}{l}\text { L'objet est } \\
\text { de raréfier } \\
\text { l'information } \\
\text { utile pour les } \\
\text { concurrents. }\end{array}$ \\
\hline $\begin{array}{l}\text { Non- } \\
\text { substitution }\end{array}$ & Loi du secteur. & $\begin{array}{l}\text { Seul moyen de } \\
\text { rester caché. }\end{array}$ & $\begin{array}{l}\text { Seul moyen } \\
\text { d'introduire de } \\
\text { la visibilité } \\
\text { dans le secteur. }\end{array}$ & $\begin{array}{l}\text { Le groupement } \\
\text { d'indépendants } \\
\text { est un substitut } \\
\text { partiel. }\end{array}$ & $\begin{array}{l}\text { En l'absence, } \\
\text { le danger à } \\
\text { l'imitation est } \\
\text { patent. }\end{array}$ \\
\hline Appropriation & $\begin{array}{l}\text { Part léonine } \\
\text { de la valeur } \\
\text { ajoutée ( } c f . \\
\text { résultat / CA). }\end{array}$ & $\begin{array}{l}\text { Résultat } \\
\text { supérieur à } \\
10 \% \text { du CA. }\end{array}$ & $\begin{array}{l}\text { Renforcement } \\
\text { des contrôles et } \\
\text { des normes par } \\
\text { les autorités } \\
\text { de tutelle. }\end{array}$ & $\begin{array}{l}\text { Acquisition } \\
\text { d'un actif de } \\
\text { qualité à un } \\
\text { prix faible. }\end{array}$ & $\begin{array}{l}\text { Baisse de la } \\
\text { réactivité des } \\
\text { concurrents aux } \\
\text { lancements de } \\
\text { produits. }\end{array}$ \\
\hline
\end{tabular}

TABleau 3

Actifs intangibles et actions grises

\begin{tabular}{|c|c|c|c|c|c|}
\hline \multirow[b]{2}{*}{ Avantage } & \multicolumn{5}{|c|}{ Ressources et compétences } \\
\hline & Fonctionnel & Culturel & Positionnel & Réglementaire & \\
\hline \multirow{2}{*}{ Facteurs humains } & & Indep & & & Savoir-faire \\
\hline & & & $\begin{array}{l}\text { Les Tilleuls } \\
\text { DBN } \\
\text { Santix }\end{array}$ & & Actifs \\
\hline
\end{tabular}

Facteurs non humains Magma

Source : À partir de Tywoniak (1998), relevé dans Hall, 1993, p. 611.

Revue internationale P.M.E., vol. 16, nos 3-4, 2003 
FiguRE 2

Le positionnement des cas étudiés

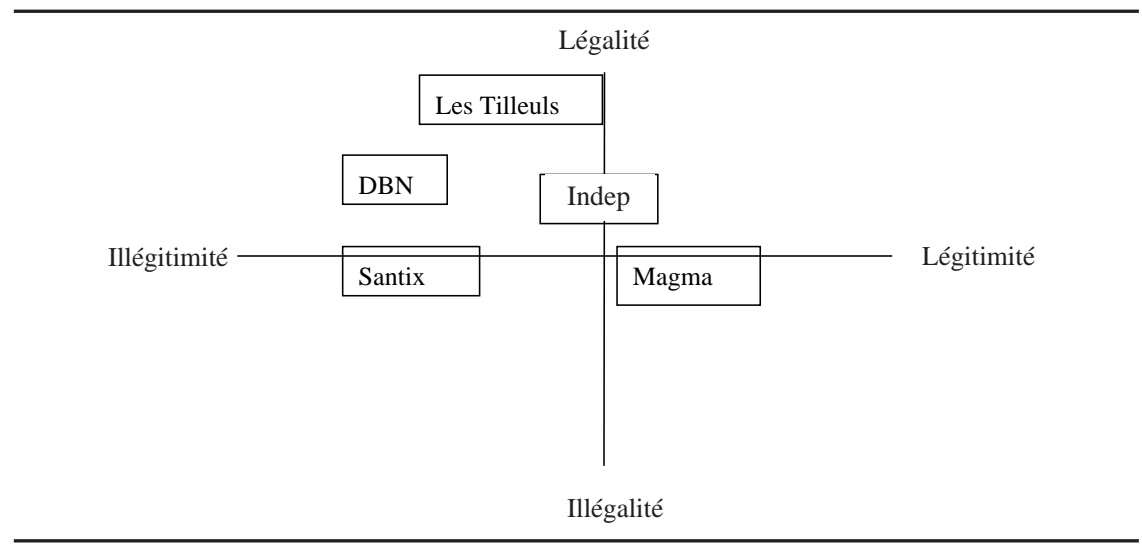

L'objectif de Magma est de tromper la concurrence, de l'influencer afin de l'orienter vers de fausses pistes. Pour ce faire, l'entreprise détourne la législation sur les brevets. Cette démarche de dissimulation des orientations technologiques de l'entreprise paraît légitime, il s'agit pour l'entreprise de se protéger. Toutefois, ce détournement de la législation sur les brevets est illégal. Mais les risques juridiques encourus pour cette pratique sont faibles, ce qui justifie son application.

La politique de DBN a été de violer les règles de la concurrence afin d'établir une entente sur les prix. Mais sa politique consiste en outre à influencer les acteurs du secteur pour qu'ils suivent la stratégie édictée par ses soins. Les acteurs qui refusent d'adopter sa politique connaissent des difficultés à travailler pour le secteur de l'automobile. Si la pratique est légale, elle n'est pas légitime d'un point du point de vue de l'éthique des affaires puisqu'il y a coercition.

Dans le cas des Tilleuls, la politique du dirigeant est parfaitement légale mais peut paraître illégitime dans ce secteur éthique, car il y a dissimulation de la véritable nature de cette entreprise, le niveau d'illégitimité est toutefois faible.

La pratique du cas Indep est légale, toutefois, elle vise à imposer sa propre légalité (manipulation de la loi, donc niveau de légalité faible) qui peut paraître, d'un point de vue du jeu des affaires, faiblement illégitime.

Dans le cas Santix, certaines pratiques peuvent sembler illégales, mais étant donné la difficulté de la preuve, le risque de sanction est faible. Du point de vue du jeu des affaires, le cas est illégitime, toutefois, la volonté à l'époque des pouvoirs publics de voir les établissements de santé se concentrer lui a donné de la légitimité au plan macroéconomique. 
In fine, les actions grises pratiquées par nos différents belligérants appartiennent à la sphère de la tentative de contrôle du jeu local des affaires ( $c f$. figure 1). En revanche, nous n'avons pas observé de pratiques de tentative d'influence, stratégie qui appartiendrait davantage à la sphère de grandes entreprises aux moyens conséquents. Un prolongement de notre étude consisterait à déterminer si les types d'actions grises mises en œuvre sont contingents à la taille des entreprises.

\section{Conclusion}

La veille stratégique a pour mission de faire entrer de l'externe dans l'interne afin que le système organisationnel puisse réagir pertinemment, notamment par des actions grises. Celles-ci permettent de lier information et action dans un registre structurant. Cette compétence repose sur une combinaison complexe de ressources. La mise à l'épreuve des critères de l'avantage concurrentiel tend à montrer que les actions grises correspondent à une compétence distinctive ou cardinale. En ce sens, elles confèrent un avantage concurrentiel.

Néanmoins, si les actions grises peuvent être une compétence permettant de changer les règles du jeu, la rupture introduite par un concurrent ou un autre acteur peut ruiner l'avantage concurrentiel de la firme. Le critère de longévité pose problème. Lorsque les règles du jeu viennent à se modifier, la situation de l'entreprise devient précaire et la compétence distinctive en matière d'actions grises devient une compétence simple qui ne fonde plus l'avantage concurrentiel. Dans ce cas, les compétences distinctives ne sont plus opérantes en tant que telles. La fonction de veille comme simple modalité d'observation de l'environnement demeure une compétence centrale dont la firme ne saurait se départir. La mission du système de veille consiste à percevoir les évolutions de l'environnement et en particulier les ruptures (Barney, 1996). La veille au sens traditionnel conserve donc une importance centrale dans les dispositifs stratégiques des entreprises.

L'analyse, centrée prioritairement sur l'interne de l'entreprise, peut faire oublier les contraintes édictées par l'environnement, notamment par la situation de marché, ou encore amener à méconnaître le comportement des concurrents avec lesquels l'organisation est en interaction.

Toutefois, la maîtrise des actions grises et l'aptitude à percevoir les mutations de l'environnement font appel à de véritables compétences organisationnelles. Il apparaît donc illusoire de penser un système de veille indépendamment de la prise en compte des compétences organisationnelles. Là réside l'apport complémentaire de la théorie des ressources. Celle-ci peut nous expliquer pourquoi un système de veille déconnecté des compétences organisationnelles peut être inopérant. En effet, l'information recueillie en externe doit trouver un écho en interne 
afin de s'adapter aux mutations de l'environnement ou afin d'imaginer, compte tenu des ressources de la firme, des actions permettant de construire avantageusement le futur.

La théorie des ressources reconnaît le caractère idiosyncrasique des compétences de l'entreprise pour les actions grises et la veille. Elle nous explique l'échec de politiques de veille qui, non reliées à des compétences internes, deviennent inutiles. Ce problème de l'ancrage organisationnel est fondamental. Également, sans but assigné au système de recueil de l'information, celui-ci se déstructure et devient alors inefficace. L'organisation est fermée aux signaux extérieurs. Or, pour fonctionner, un système, quel qu'il soit, doit avoir un minimum d'ouverture sur l'extérieur (Lapierre, 1993).

\section{Bibliographie}

Alliaume, M., R. Condor et S. Grandval (1998), Étude sur la pratique de l'intelligence économique et des besoins en information des entreprises, CCI/Industria Caen, mars.

ARRÈGLE, J.L. et B. QUÉLIN (2001), «L'approche fondée sur les ressources », dans A.C. Martinet et R.A. Thiétart, Stratégies, Actualité et futurs de la recherche, Paris, Vuibert, p. 273-287.

Azzone G., U. Bertelè et A. RANGONE (1995), «Measuring resources for supporting resource-based competition», Management Decision, vol. 9, p. 57-62.

BARNEY, J. (1991), «Firm resources and sustained competitive advantage », Journal of Management, vol. 17, $\mathrm{n}^{\circ}$ 1, p. 99-120.

BARNEY, J.B. (1995), «Looking inside for competitive advantage», Academy of Management Executive, no 4, p. 49-61.

BARNEY, J.B. (1996), «The resource-based theory of the firm», Organisation Science, vol. 7, $\mathrm{n}^{\circ}$ 5, septembre-octobre, p. 469.

BAUMARD, P.H. (1991), Stratégie et surveillance des environnements concurrentiels, Paris, Masson.

Baumard, P.H. (1996), Prospective à l'usage du manager, Paris, Litec.

BAUMARD, P.H. (1997), «L'information stratégique dans la grande organisation », Systèmes d'information et management, vol. 2, no 2, p. 3-25.

CLERC, P.H. (1995), «Intelligence économique », Paris, Encyclopædia Universalis, p. 195.

Collis, D.J. et C.A. MonTGomery (1995), «Competing on resources : strategy in the 1990s », Harvard Business Review, juillet-août, p. 118-128.

Cova, B. et R. SALLE (1999), Le marketing d'affaires, Paris, Dunod.

DAVID, A. (2000), «Logique, épistémologie et méthodologie en sciences de gestion : trois hypothèses revisitées », dans A. David, A. Hatchuel et R. Laufer, Les nouvelles fondations des sciences de gestion, Paris, Vuibert, p. 83-109.

Revue internationale P.M.E., vol. 16, nos 3-4, 2003 
DURAND, T. (2000), «L'alchimie de la compétence », Revue française de gestion, janvierfévrier, p. 84-101.

DurieuX, F., M. GIROD-SEVILlE et V. PERret (2000), «La planification stratégique à la complexité », L'Expansion Management Review, septembre, p. 82-91.

GRANDVAL, S. (2000), Dynamique des actions grises dans l'intelligence concurrentielle, Thèse de doctorat de l'Université de Caen.

GRANDVAL, S. et R. SOPARNOT (2002), «La précurrence : la traduction des stimuli externes en action pertinente», La Revue des sciences de gestion, Direction et Gestion, vol. 37, no 195, mai-juin, p. 41-52.

HALL, R. (1993), « A framework linking intangible resources and capabilities to sustainable competitive advantage », Strategic Management Journal, vol. 14, no 8, p. 607-618.

LAPIERRE, J.W. (1993), L'analyse des systèmes, l'application aux sciences sociales, Paris, Syros.

LESCA, H. (1994), Veille stratégique, l'intelligence de l'entreprise, Lyon, Aster.

MARTINET, A.C. (1996), «L'entreprise et la stratégie : l'évolution de la pensée stratégique », Les cahiers français, La Documentation française, mars-avril, nº 275.

MARTinet, A.C. (1998), «Les jeux du management stratégique formes et figures élémentaires », dans J. Thépot, Gestion et théorie des jeux, Paris, Vuibert.

MARTINET, B. et Y.M. MARTI (1995), L’intelligence économique, les yeux et les oreilles de l'entreprise, Paris, Les Éditions d'Organisations.

MARTRE, H. (1994), Intelligence économique et stratégie des entreprises, Paris, Commissariat général du plan, La Documentation française.

Miles, B. et M. Huberman (1991), Analyse des données qualitatives : Recueil de nouvelles méthodes, Bruxelles, De Boek Université.

MintzBerg, H. (1982), Structure et dynamique des organisations, Paris, Éditions d'Organisations.

NAlebufF, B. et A. BRANDEnburger (1996), La coopétition, une révolution dans la manière de jouer concurrence et coopération, Paris, Village mondial.

SpANOS, Y.E. et S. LiOUKAS (2001), «An examination into the causal logic of rent generation : contrasting Porter's competitive strategy framework and the resourcebased perspective », Strategic Management Journal, vol. 22, n 10, p. 907-934.

TeEce, D.J., G. PISANO et A. ShuEN (1997), « Dynamic capabilities et strategic management », Strategic Management Journal, vol. 18, n 7, p. 509-533.

TYWONIACK, S.A. (1998), «Le modèle des ressources et des compétences : un nouveau paradigme pour le management stratégique?», dans H. Laroche et J.-P. Nioche, Repenser la stratégie, Paris, Vuibert, p. 166-204.

Usunier, J.-C. et G. VERNA (1994), La grande triche, Paris, La Découverte.

WACHEUX, F. (1993), Épistémologie et sciences de gestion, Paris, Economica.

WERnERFELT, B. (1984), «A resource-based theory of the firm », Strategic Management Journal, vol. 5, no 1, p. 99-120.

YIN, R.K. (1994), Case Study Research, Design and Methods, Londres, Sage Publications.

Revue internationale P.M.E., vol. 16, nos 3-4, 2003 